\title{
La bibliothèque de Solesmes : prières et études
}

\author{
Père Bertrand Gamelin
}

\section{(2) OpenEdition}

Journals

Édition électronique

URL : http://journals.openedition.org/rbnu/914

DOI : 10.4000/rbnu.914

ISSN : 2679-6104

\section{Éditeur}

Bibliothèque nationale et universitaire de Strasbourg

\section{Édition imprimée}

Date de publication : 1 mai 2017

Pagination : 70-73

ISBN : 9782859230661

ISSN : 2109-2761

\section{Référence électronique}

Père Bertrand Gamelin, "La bibliothèque de Solesmes : prières et études », La Revue de la BNU [En ligne], 15 | 2017, mis en ligne le 01 novembre 2019, consulté le 11 décembre 2020. URL : http:// journals.openedition.org/rbnu/914; DOI : https://doi.org/10.4000/rbnu.914

\section{(C) $10(0$}

La Revue de la BNU est mise à disposition selon les termes de la Licence Creative Commons Attribution - Pas d'Utilisation Commerciale - Partage dans les Mêmes Conditions 4.0 International. 


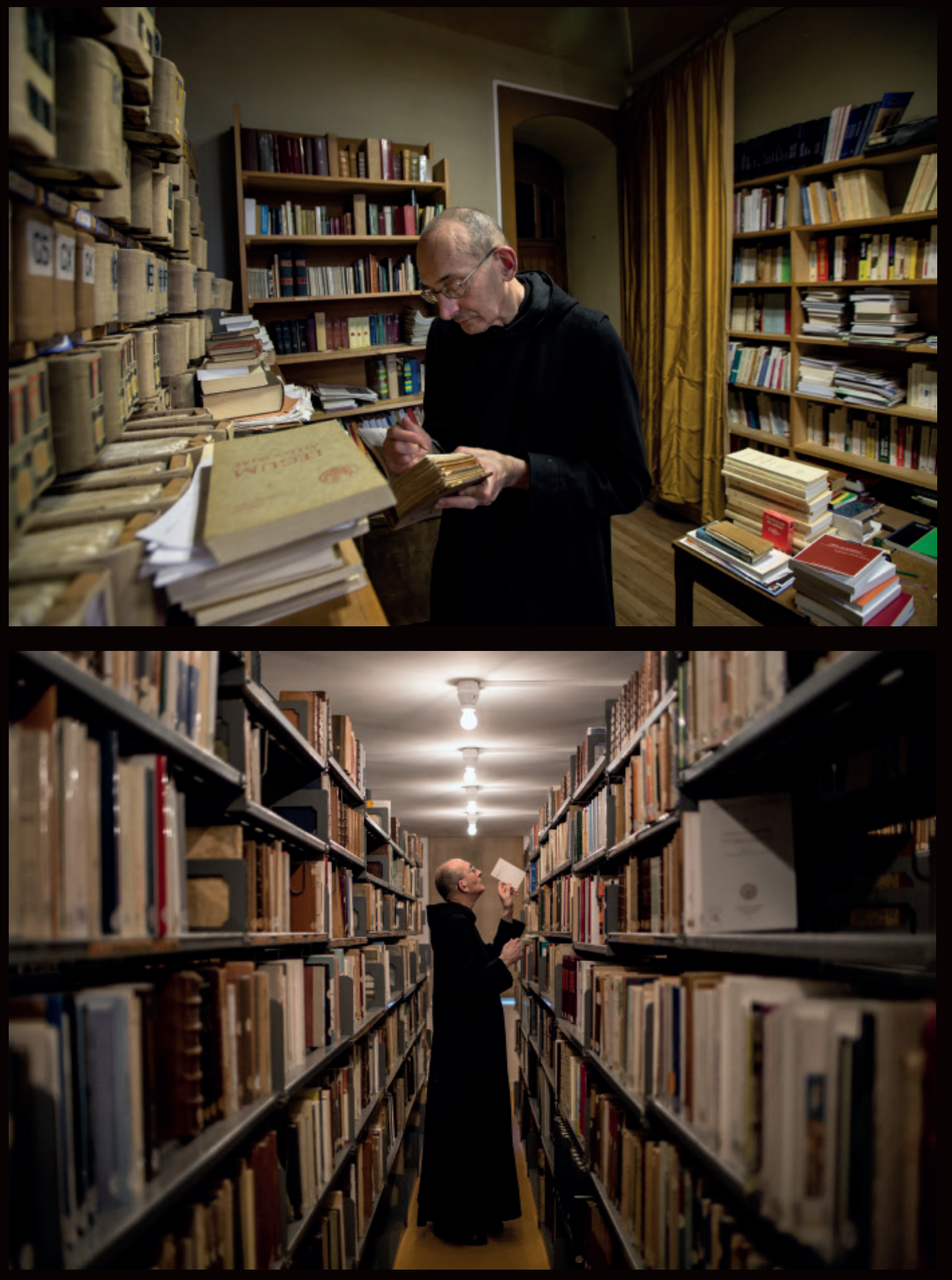




\section{LA BIBLIOTHÈQUE DE SOLESMES : PRIÈRE ET ÉTUDES \\ PAR LE PÈRE BERTRAND GAMELIN}

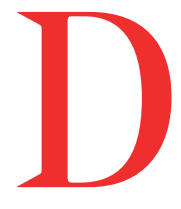

om Guéranger, qui restaura la vie bénédictine en France à Solesmes en 1833, voulait, disait-il, fonder « une maison de prière et d'études ». Prière et études sont en effet une des caractéristiques principales du monachisme bénédictin. Saint Benoît prévoit une lecture à la table des moines. Elle ne doit jamais y manquer. Un livre est donné le premier jour du carême, qui doit être lu intégralement. Chaque jour, un temps important est consacré à la « lectio divina ». Pourquoi ? Parce que l'esprit doit se nourrir de la parole de Dieu. En effet, comme dit l'Évangile : "L'homme ne vit pas que de pain, mais de toute parole qui sort de la bouche de Dieu ». La raison d'être de cette "lectio divina » est bien exprimée par saint Isidore : "L'homme parle à Dieu par la prière, par la lecture c'est Dieu qui parle à l'homme ". Quels sont les livres que recommande saint Benoît ? L'Écriture sainte, bien sûr, et les « livres des saints pères catholiques " qui nous enseignent le moyen de parvenir à Dieu. Saint Benoît fait une référence explicite à saint Jean Cassien et à saint Basile. Par la suite, les monastères bénédictins, en particulier sous l'influence de Cassiodore, chercheront à rassembler dans leurs bibliothèques les chefs-d'œuvre de l'esprit humain ${ }^{1}$. Bien que le quatrième concile de Carthage ait ordonné «que l'évêque ne lise pas les livres des païens ", les moines auront chez eux les grands classiques de la littérature grecque et romaine. Au 17 siècle, Dom Mège écrira ce passage délicieux : " Les bibliothèques sont parmi les choses les plus précieuses et les plus nécessaires aux religieux... Nos bibliothèques sont nos trésors qui enferment avec toutes les sciences et tous les arts, les plus innocentes et les plus chères délices des Solitaires ». On peut résumer cela en disant que les moines, qui recherchent la sainteté, ne veulent pas négliger la sainteté de l'intelligence.
Dom Guéranger était bien conscient de tout cela. Quand il réussit à racheter Solesmes, l'ancienne bibliothèque du prieuré fondé vers 1010 avait été vendue à la Révolution. Il fallait partir de zéro. Pour un millier de francs, l'abbé Guéranger put obtenir des exemplaires en double de la bibliothèque du grand séminaire du Mans. Au total, 600 volumes, dont la collection des conciles de Labbe. Passionné par les livres, l'abbé Guéranger faillit ruiner sa jeune communauté, en achetant pour 1200 francs la collection des Bollandistes (Acta sanctorum). Cet achat eut lieu alors qu'il n'avait en mains que le pain de quelques jours. 53 volumes furent expédiés à Sablé et arrivèrent à l'abbaye dans un tombereau traîné par quelques mules. Peu après, l'abbé Guéranger achetait les œuvres complètes de saint Bonaventure, qu'il aimait particulièrement. Sa passion pour les livres se manifesta encore par l'achat de la vie des papes d'Anastase le Bibliothécaire. Le livre fut apporté à Notre-Dame du Chêne, à trois kilomètres de l'abbaye, et Dom Guéranger le porta sur son dos jusqu'au monastère. Il dut s'arrêter plus de dix fois. Par la suite, Dom Guéranger ne cessa d'enrichir la bibliothèque, en particulier en se faisant offrir de nombreux exemplaires de bréviaires et de missels, et ceci pour écrire ses fameux livres sur les Institutions liturgiques, dans lesquels il défend la liturgie romaine et critique, souvent sévèrement, les liturgies gallicanes. Il acheta ou se fit offrir également de nombreux livres de patristique et d'histoire de l'Église pour aider au travail des moines. Il bénéficia de nombreux dons du gouvernement, de l'École des chartes, mais aussi de collectionneurs privés. Citons, par exemple, le cas de l'érudit tourangeau Étienne Cartier, qui lui offrit des manuscrits que l'on ne s'attendrait pas à trouver dans une abbaye. Ce sont des œuvres de Claude de SaintMartin, le fameux " théosophe inconnu ", et même de Martinez de Pasqually, un mystérieux cabaliste. D'autres moines obtinrent des cadeaux analogues. Un 
des moines de Dom Guéranger, le futur cardinal Pitra, lors d'un voyage d'études en Russie, se fit offrir par un colonel polonais qui résidait à Moscou des livres liturgiques de l'Église orthodoxe. Il faut ajouter que ce même Dom Pitra avait la fâcheuse habitude de ne pas rendre les manuscrits précieux qu'on lui prêtait. On l'avait surnommé plaisamment « Dom Pirate ». Mentionnons encore un curé de Pau, dans le Béarn, qui offrit à Dom Guéranger l'antiphonaire de Leyre du $12^{\mathrm{e}}$ siècle. Il faut ici avouer que cet antiphonaire avait été volé en Espagne par les soldats de Napoléon. Dom Guéranger bénéficia aussi de cadeaux d'écrivains contemporains. C'est ainsi que son ami Louis Veuillot, le célèbre journaliste, lui offrit presque toutes ses œuvres. Le futur cardinal Newman fit de même avec une des siennes, lors d'un voyage de l'abbé en Angleterre. Disons tout de suite qu'il y a là une tradition qui a perduré dans l'histoire de Solesmes. C'est ainsi que Huysmans offrit un exemplaire de La Cathédrale. Et comment ne pas mentionner la plaisanterie de Paul Valéry, offrant un de ses livres à l'abbaye "pour être mis à l'enfer " ? Grâce à toutes ces acquisitions, la bibliothèque de Solesmes qui, vers la fin de 1833, comptait déjà 4000 volumes, en avait 12000 en 1857. L'abbaye étant pauvre à l'époque, les dons l'emportent sur les achats.

La bibliothèque de l'abbaye continua à s'enrichir après la mort de Dom Guéranger, en 1875. Mais en 1880 et 1881, les moines furent expulsés de leur abbaye, leur congrégation n'étant pas reconnue par le gouvernement. La bibliothèque suivit le sort des moines : on la regroupa, comme on put, dans les maisons du village de Solesmes. On disait que, pour aller de la liturgie au droit canon, il fallait traverser la rue. Ce n'étaient pas des conditions très favorables pour l'étude. Les moines purent rentrer chez eux à partir de 1895 , et réinstallèrent leur bibliothèque. Mais en 1901, ce fut le grand exil. En effet, le père abbé Dom Delatte n'avait pas voulu demander l'autorisation au gouvernement, sachant que, de toute façon, elle serait refusée : « Nous n'avons pas à demander l'autorisation, car nous sommes autorisés par la loi divine et la loi naturelle ». Les moines partent en Angleterre. La bibliothèque les accompagne, dans des centaines de caisses. Certaines d'entre elles ne seront pas ouvertes. En effet, les moines pourront revenir en 1922 dans leur abbaye. Et une nouvelle bibliothèque est construite de 1936 à 1938. Elle sera agrandie après la Seconde Guerre mondiale.
Aujourd'hui, on peut estimer à 200000 livres le contenu de la bibliothèque. Que peut-on y trouver? Tout ce qui sert à la « lectio divina », notamment les éditions des pères de l'Église, et particulièrement les beaux livres que nous ont légués les mauristes ; des livres liturgiques, qui rappellent l'importance du rôle de Solesmes dans le mouvement liturgique ; une collection très complète des textes des conciles et des papes, car les moines de Solesmes ont toujours eu à cœur d'étudier les sources de la doctrine et l'enseignement autorisé du magistère romain. Naturellement, on y trouve aussi un fonds important de mystique et de spiritualité. Signalons en outre beaucoup de livres d'histoire de l'Église, car après la Seconde Guerre mondiale, des moines ont donné des recensions pour la Revue d'histoire ecclésiastique. Nous tenons également à avoir beaucoup de livres de théologie et de philosophie et, en particulier, les œuvres de saint Thomas d'Aquin. Mais on ne trouve pas que des livres religieux à la bibliothèque de Solesmes. On y trouve tout ce qui met en relief le Beau, le Bien et le Vrai - par exemple, beaucoup de livres d'art et d'histoire profane. La collection de livres d'histoire de France est elle aussi très importante. Signalons en particulier un très grand nombre de livres sur Jeanne d'Arc, dont beaucoup viennent de la bibliothèque d'une figure contestée, l'abbé Jouïn, curé de Saint-Augustin à Paris. Fidèles à l'enseignement du concile Vatican II, les moines ont cherché à élargir leurs préoccupations intellectuelles. C'est ainsi que le fonds d'histoire des religions a été considérablement augmenté ces dernières années.

Enfin, il y a la musique. Nous n'en parlons pas ici en détail, car tous les documents concernant le chant grégorien sont conservés à la «Paléographie musicale", service différent de celui de la bibliothèque. Cette "Paléographie » est célèbre par sa collection de photographies des manuscrits de chant grégorien. Depuis la fin du $19^{\mathrm{e}}$ siècle jusqu'à l'entre-deux-guerres, plusieurs moines entreprirent en effet un grand périple à travers toutes les bibliothèques d'Europe. Certaines de ces photographies sont d'autant plus précieuses qu'elles sont le témoignage de manuscrits détruits pendant les guerres. Le cas le plus célèbre est celui dit du « Graduel de Chartres ", la bibliothèque de cette ville ayant été détruite pendant le dernier conflit mondial. 


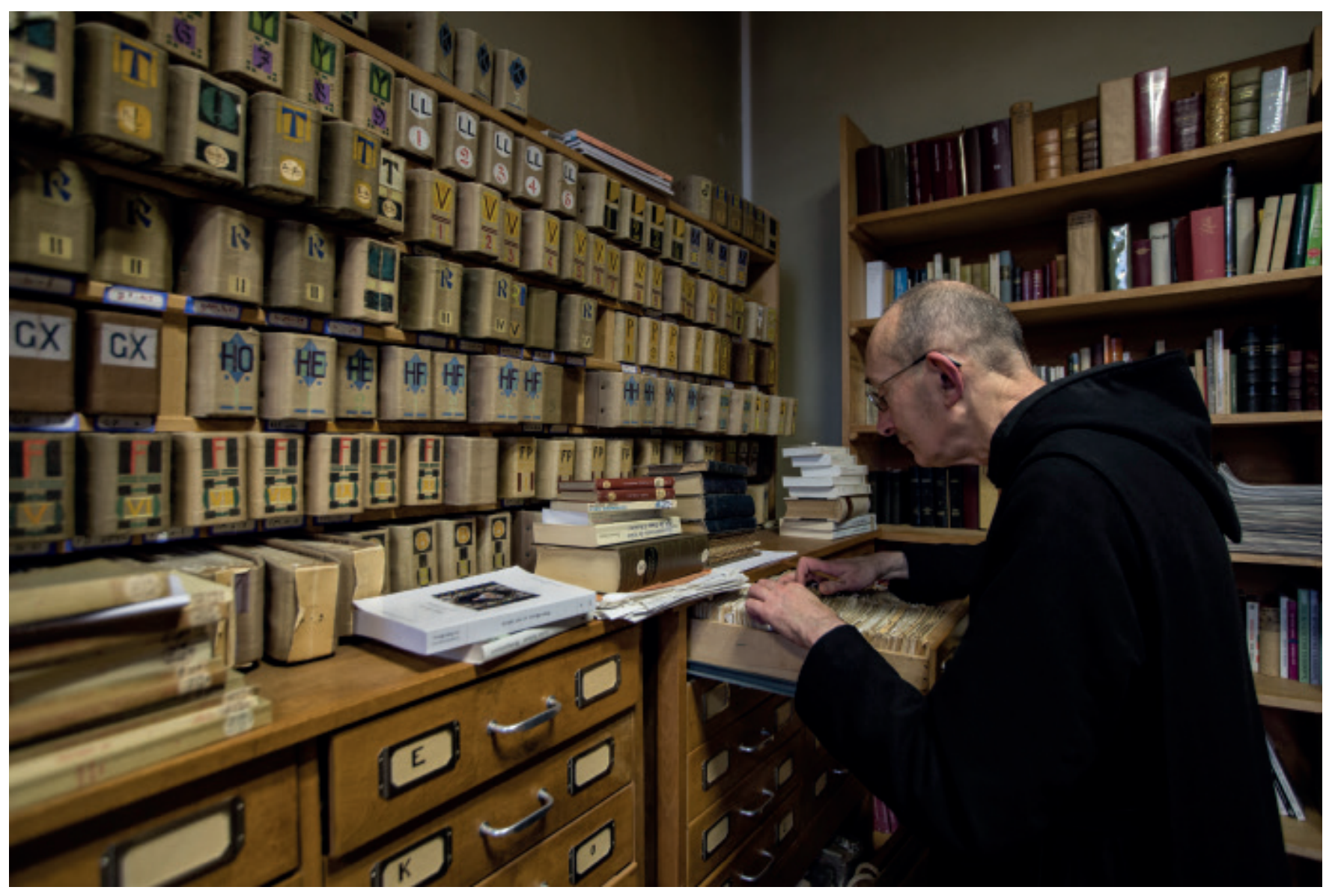

Nous avons donc vu ce qu'on peut trouver dans la bibliothèque de Solesmes. Bien sûr, elle est faite d'abord pour les moines, et ceux qui parmi nous sont chargés d'un cours ou font des études de théologie l'utilisent beaucoup. Pour les moines, l'accès à la bibliothèque est entièrement libre (mettons à part le cas du noviciat, qui a sa bibliothèque particulière) et on peut y aller jour et nuit ! Le système de classification a été élaboré après la Seconde Guerre mondiale. C'est un système " maison ", qui n'a rien à voir avec la classification internationale. Mais l'informatisation aide beaucoup la consultation, par exemple dans des cas comme celui des livres sur le judaïsme qui sont dispersés en plusieurs endroits différents. Mais la bibliothèque n'est pas faite que pour les moines. Elle peut accueillir des chercheurs, avec cette réserve qu'il n'y a pas vraiment de "salle d'études ", et que le temps du bibliothécaire est limité. Les demandes peuvent être de toute sorte : n'avons-nous pas eu une fois celle d'un écrivain désireux d'entreprendre une " histoire de la sardine " ? Il fut fort satisfait de l'envoi de textes d'Aristote sur le sujet !
Nous avions commencé cet article par l'évocation de la figure de Dom Guéranger amenant des livres sur une carriole. Les moyens techniques se sont améliorés, mais l'esprit reste le même : l'amour de la parole de Dieu, et le désir de le communiquer à d'autres. Les moines attachent beaucoup d'importance aux études, mais toujours en vue de la prière et de la contemplation. Une anecdote pour terminer : le jeune Paul Delatte, futur abbé de Solesmes, vint rendre visite à Dom Guéranger, alors qu'il n'était qu'un jeune prêtre. Il pensait déjà à la vie monastique, mais voyait surtout dans le monastère un lieu d'études savantes. Dom Guéranger lui répondit : «Monsieur l'abbé, le bénédictin, avant d'être un homme d'études, est l'homme de la prière sociale de l'Église ». Le jeune Paul Delatte ne comprit pas tout de suite cette phrase. Il comprit plus tard, et il revint.

\section{NOTE}

1- Voir en particulier, à ce sujet, l'article de Robert Bedon p. 9 